\title{
REPORT ON GIANT NERVE FIBRES IN NANOMIA
}

AUTHOR(S):

Mackie, G. O.

\section{CITATION:}

Mackie, G. O.. REPORT ON GIANT NERVE FIBRES IN NANOMIA.

PUBLICATIONS OF THE SETO MARINE BIOLOGICAL LABORATORY 1973, 20: 745-756

ISSUE DATE:

1973-12-19

URL:

http://hdl.handle.net/2433/175745

RIGHT: 


\title{
REPORT ON GIANT NERVE FIBRES IN NANOMIA
}

\author{
G. O. MACKIE \\ Department of Biology, University of Victoria \\ Victoria, B. C., Canada \\ With 8 Text-figures
}

\begin{abstract}
The siphonophore Nanomia has a pair of giant fibres running along the stem, the larger of which conducts at velocities up to 3 metres/second. Histologically the two giant fibres appear to be composite, partially syncytial structures representing specializations within the general ectodermal nerve plexus.

Electrical events are through-conducted independently in the two fibres. Both systems excite the stem musculature. Conduction velocities are proportional to fibre diameters. Conduction occurs in both directions in each giant fibre. An absolute refractory period of 5-7 milliseconds is observed. The two systems are spontaneously active, showing distinctive pulse patterns.

Intracellular recordings with glass microelectrodes from the larger giant fibre show conventional action potentials of about 60 millivolts. Addition of tetrodotoxin to the outside medium at $10^{-6} \mathrm{~g} / \mathrm{ml}$ does not affect spike production.

Palpon tentacles have two chains of small neurones. Two conduction systems, one faster than the other, can be shown physiologically in the tentacles. This may be taken as a simplified model for the stem. The existence of two synergistically acting fast conducting systems is interpreted in relation to gradation of response intensity with distance from the site of stimulation.
\end{abstract}

Giant axons serve as rapid conduction pathways in the escape behaviour of many invertebrates. No such system has yet been described in a coelenterate, although some medusae have a giant fibre nerve net which conducts at velocities up to $0.5 \mathrm{~m} / \mathrm{sec}$, spreading impulses to the swimming muscles during locomotion (BULLOCK and HORRIDGE, 1965). The rapidly conducting mesenteric nerve net of some sea anemones mediates a protective response and is therefore functionally comparable to giant fibre systems in other invertebrates, but the axons comprising it are quite small.

The present report deals with a giant fibre system in a siphonophore, Nanomia cara. The system is interesting for the comparisons it permits with the giant fibre systems of other phyla and for the opportunity it affords to study neuromuscular interaction at the cellular level, a virtually unexplored field in coelenterates. SPENCER (1971) showed that intracellular recordings could be obtained from Nanomia epitheliomuscular cells. I have made similar recordings and have made some progress in correlating intra-muscular potentials with neural events, but this work is not ready for presentation at this time. 


\section{Materials and Methods}

Nanomia were collected from the dock at the University of Washington Laboratories at Friday Harbor, U.S.A., and were kept in tanks in the laboratory by methods described previously (MACKIE and BOAG, 1963; MACKIE, 1964).

Plastic suction electrodes were used for stimulating the stem and for recording extracellularly. For intracellular recordings glass microelectrodes in the 30 to 50 megohm range were employed. A Vickers Stereo II microscope with paired $7.5 \mathrm{x}$ water immersion objectives was used for positioning the microelectrodes.

For histological study, methylene blue staining was employed in conjunction with phase contract microscopy. Material for electron microscopy was prepared by standard methods using epon embedding, and material from some of the blocks was cut at $1 \mu$ for optical study.

Where the object was to compare conduction velocities with fibre diameters, pieces of stem were relaxed in a mixture of sea water and isotonic magnesium chloride in the proportion 1:7.5. This concentration does not affect nervous conduction appreciably, but it raises the muscle response threshold to the point where the stimulation caused by attachment of electrodes and giving infrequent low voltage shocks results in no visible contraction of the stem. The recording electrodes were then moved apart pulling the stem into a straight line without stretching it. After conduction velocities had been established over a given length of the stem, the piece in question was put into 1:1 sea water-magnsium mixture, cooled to near $0^{\circ} \mathrm{C}$ and fixed while being held by forceps in the extended condition. Values for fibre diameters were based on typical sections taken from several points along the stem in regions over which velocities had been measured. Since the shapes of the axons in cross section rarely approached the perfect circle, the areas of the axons were measured from photographs, using a planimeter, and the equivalent perfect circles were calculated form which diameter values were obtained. Conduction velocities taken at different times were done within the temperature range $13.0-16.5^{\circ} \mathrm{C}$. For comparative purposes (e.g. Fig. 4) temperature corrections were applied, based upon a $\mathrm{Q}_{10}$ value of 2.0 established for conduction velocities over the 10 to $20^{\circ} \mathrm{C}$ range.

\section{Results}

When the stem of a Nanomia is stimulated it contracts either locally or along its length and at the same time the animal swims forward by concerted action of the swimming bells, a response shown to involve transmission through a nerve tract from the stem to each swimming bell (MACKIE, 1964). Both stem contraction and swimming can now be shown to depend upon rapidly conducted events, recordable with external electrodes from the stem surface, which represent impulses in a pair of giant nerve fibres. 


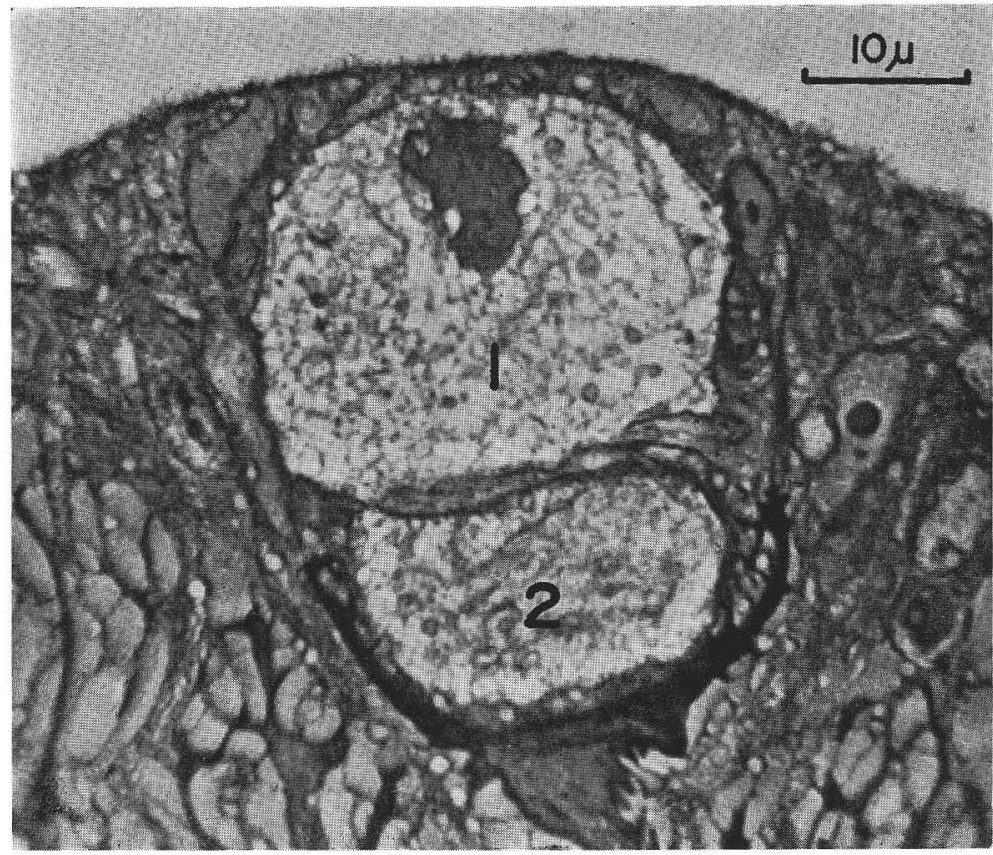

Fig. 1. The two giant fibres ( 1 and 2$)$ in a $1 \mu$ epon section. A nucleus is present in giant fibre 1 . The fibres lie in the ectoderm and are separated from the outside by a thin layer made up of processes of ectoderm cells.

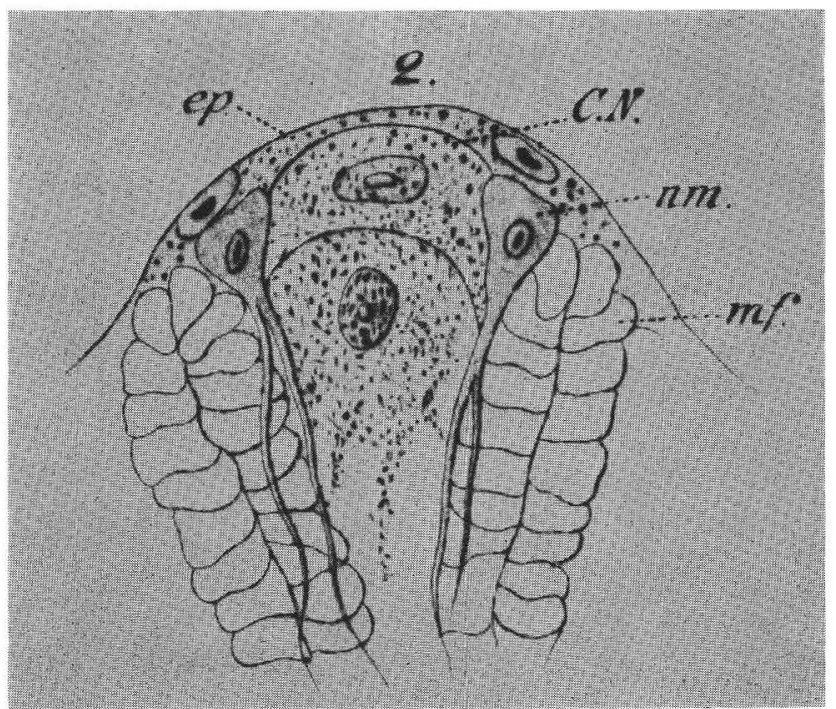

Fig. 2. KoROTNEFF's drawing of a section through the dorsal midline of the stem of Halistemma rubrum, a close relative of Nanomia cara. The two giant fibres, one of which is labelled C. N. (Central Nervous System) are clearly shown. KorotnefF thought that there were distinct "neuro-muscular cells" ( $\mathrm{n} \mathrm{m}$ ) as well as muscle fibres (m f) but this is erroneous. Both are components of epithelio-muscular cells. (Retouched from KorotnefF, 1884, Taf. 14, Fig. 2.) 
The giant fibres lie in the ectoderm along the dorsal midline of the stem. There are two of them, the outer being typically about 1.4 times larger than the inner (Fig. 1). They were first observed (Fig. 2) by KorotnefF (1884) and later by SCHNEIDER (1892), who correctly identified them as nervous structures, but these early reports were refuted by SCHAEPPI (1898) and have since been virtually ignored. (This history is reminiscent of the discovery of giant fibres in squid by L.W. WILLIAMs in 1909, but WILLIAMs' account was not refuted: it was merely overlooked.)

The axons are tubular structures with elongated nuclei scattered along them, somewhat resembling striated muscle fibres in this respect. The nuclei are usually less than $10 \mu$ in diameter, while the fibre may be up to $30 \mu$, so the nuclei do not cause bulges in the fibre. In other words there are no "cell bodies" in the structure. The structure appears syncytial in whole mounts and this appearance is largely borne out by study of sectioned material by optical and electron microscopy. In many sections, two nuclei appear without intervening cell membranes. However discontinuities do occur in the giant fibre suggestive of dichotomy or fusion, and it is unlikely that the fibre is a perfect syncytium from end to end, although functionally it behaves like one in respect to through conduction. As in the large fibre net (closed system) of Velella (MACKIE, 1960) the impression is one of a syncytial system, but one in which fibres can touch without necessarily fusing. According to ScHneIdER's account (1892), the median dorsal giant fibres in Halistemma and Forskalia are specialized portions of a more generally distributed ectodermal nerve net. SCHNEIDER was one of the best practitioners of the osmic-acetic maceration technique, and his general findings can probably be accepted although in matters of detail, e.g. interconnections between the two giant fibres his account may require correction. In Nanomia I have confirmed the existence of the ectodermal nerve net, using several different techniques, but the nature of the connections between the net and the giant fibres is still not clear to me. The net resembles the closed system of Velella and may, like it, be partially syncytial. The relationship of the median giant fibres to the net requires more study, but accepting SCHNEIDER's evidence, one may think of the giant fibres provisionally as thickened, condensed, logitudinally orientated portions of the diffuse net. Dr. E. R. RoBson suggested to me that if the Velella closed net were to be taken and stretched out in one direction and if the cytoplasm were then to be redistributed within the syncytium to provide for thickened central tracts, the result would be something like the system in Nanomia. In fact, from the scanty evidence available, the median giant system in Forskalia accords quite closely with this concept, Nanomia rather less so because the median elements have undergone a greater degree of development and thickening and are not so obviously differentiations within a net. There may be an "evolutionary series" within the physonectid siphonophores showing "stages" in the specialization of giant fibres.

An unsolved problem in the histological realm concerns the way in which the general net is differentiated into two functionally independent components. This is 
virtually required by the physiological evidence (p. 752), but cannot be verified yet on the morphological level.

Electrical stimulation of the stem reveals the presence of two fast conducting systems, one of which is faster than the other. The two fast systems $\left(\mathrm{F}^{1}\right.$ and $\left.\mathrm{F}^{2}\right)$ are present in all specimens (Fig. 3). The electrical signals characteristic of the two can usually be distinguished by wave form differences in any given preparation, though these are not consistent from one specimen to another. The difference in conduction velocity between the two is consistently observed and serves as the main key to separating them. Single shocks evoke single pulses or pulse trains in one or both systems. All pulses are through-conducted along the entire length of the stem. Both systems are spontaneously active and their output can be identified by their characteristic, highly

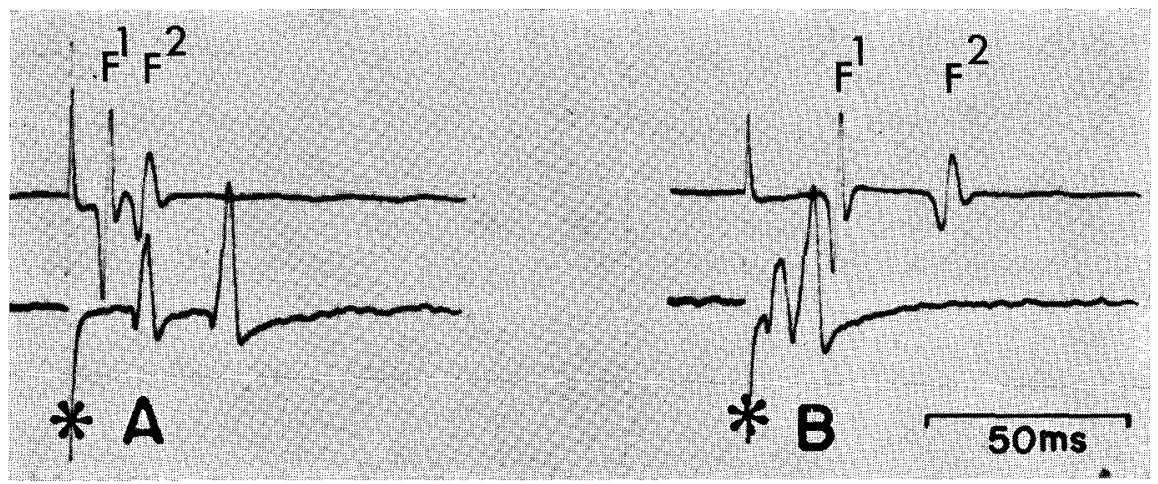

Fig. 3. Two-channel suction electrode recordings made with a stimulating electrode and two recording electrodes placed in a row along the stem. In A conduction is up the stem, in B down it. The shock artefact is marked by an asterisk. In each record a rapidly propagated event $\left(\mathrm{F}^{1}\right)$ is followed by a less rapid one $\left(\mathrm{F}^{2}\right)$. The two events are distinguishable in these recordings by their wave forms as well as by their conduction velocities but this is not always the case.

regular and often very dissimilar rhythmicities and burst patterns. The two systems can conduct in either direction (Fig. 3) and appear to do so in the normal, intact animal. They can fire repeatedly at high frequencies. The absolute refractory period is between 5 and 7 milliseconds within the temperature range observed $\left(13-16.5^{\circ} \mathrm{C}\right)$.

The two systems are functionally independent. Activity in one does not spread to the other nor do events in one trigger or inhibit events in the other.

Evidence that the two fast conducting systems are the two giant fibres has been obtained in two main ways. Conduction velocities in the two fast systems were measured for five specimens, which were then fixed and embedded and their giant fibre diameters ascertained by the method described on page 746. Plotting $\mathrm{F}^{1}$ and $\mathrm{F}^{2}$ velocities against the diameters of the two giant fibres in the same specimens gives the graph shown in Fig. 4. It will be seen that velocities are well correlated with fibre diameters. 


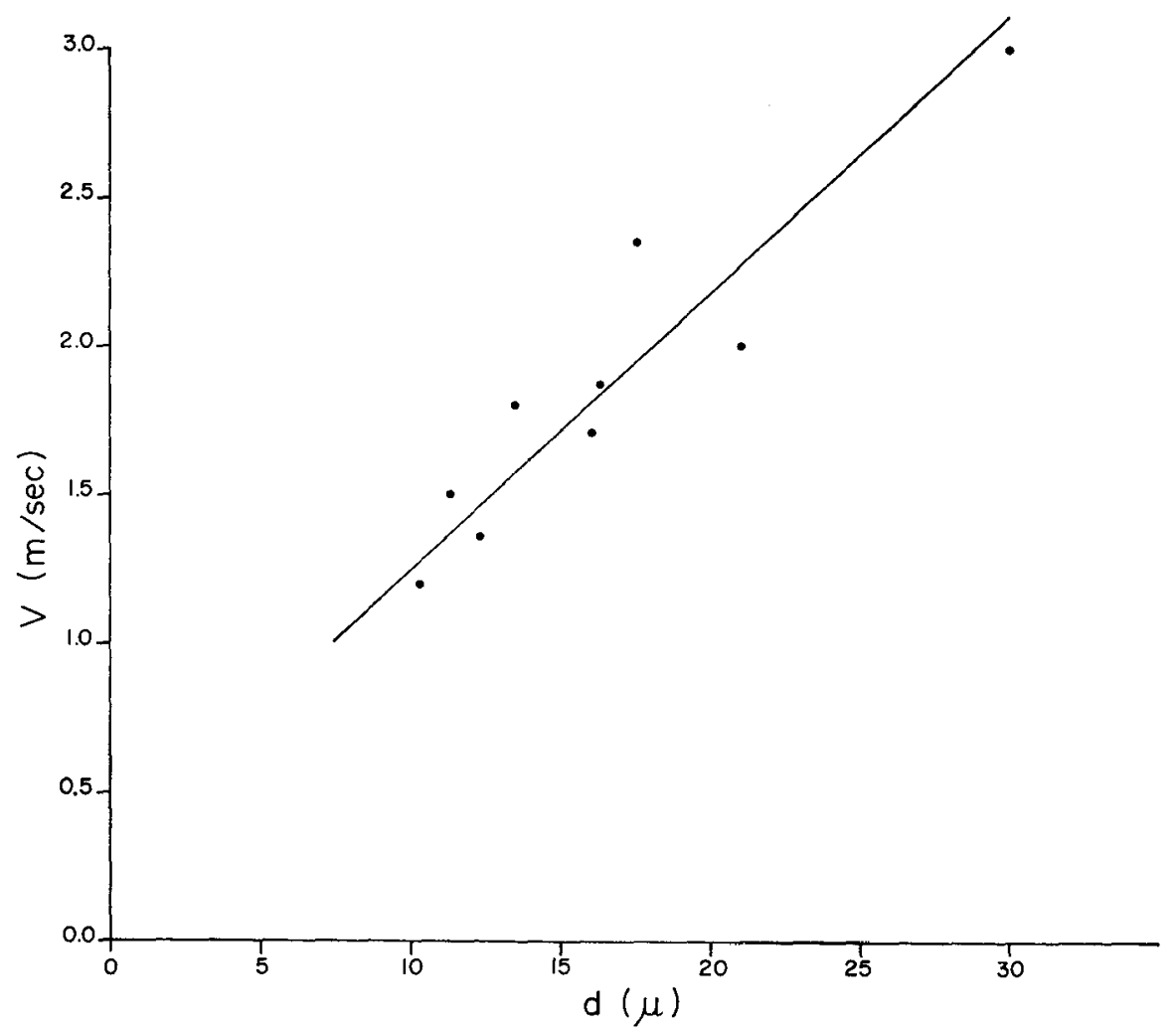

Fig. 4. Conduction velocities (V) plotted against fibre diameters (d) in Nanomia.

Another approach was to use microelectrodes to probe the region of the giant fibres and to compare signals obtained from this region with signals obtained from other regions on either side. Such experiments show that primary $F^{1}$ and $F^{2}$ events (monitored by an external suction electrode) are recorded by microelectrodes only when the electrode tip is inserted into the immediate vicinity of the giant fibres, as observed down the microscope. In most of these experiments, the signal is a positivegoing spike resembling an attenuated, intracellular action potential and rising from a more or less steady negative potential of variable magnitude. These are interpreted as partial penetrations into the giant fibres, or placements where the electrode was in a fold of the fibre without being properly in it. In some cases, the electrode appeared to have penetrated but then to have come out again, so that the initial large resting potential and action potentials became attenuated or were lost. While such recordings are unsatisfactory as examples of biophysical technique, they certainly implicate the two giant fibres in conduction of the $\mathrm{F}^{1}$ and $\mathrm{F}^{2}$ signals. Recordings in the epitheliomuscular tissue on either side show a much larger resting potential and a different set of transient depolarizations (c.f. SPENCER, 1971). 
Amongst many "near misses" from both giant fibres I have a number of examples of what appear to be good penetrations into the larger of the two giant fibres. Fig. 5 is such a record. The event is recognizable as a conventional nerve action potential of about 60 millivolts rising from a stable resting potential of about 40 millivolts. There is a small, negative-going after-potential ( $<10$ millivolts). In all such records, the electrode has picked up only the characteristic $\mathrm{F}^{1}$ signals and has failed to register $\mathrm{F}^{2}$ signals or muscle events occurring during the time it was in place.

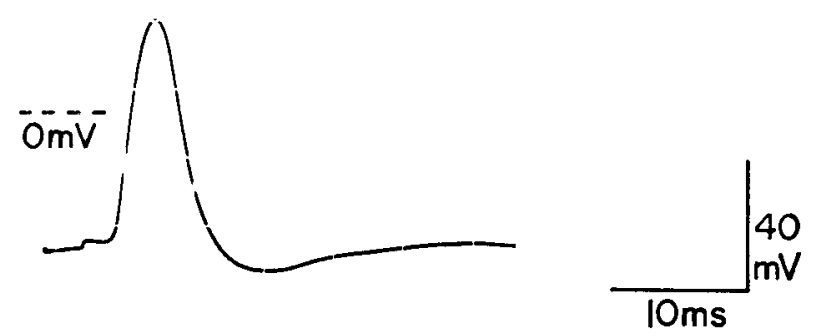

Fig. 5. Positive-going spike recorded intracellularly from giant axon 1 with a glass microelectrode.

Patton and Passano (1972) report intracellular recordings from Cyanea neurones showing composite pulses of variable amplitude and wave form and variable resting potentials. Depolarization or hyperpolarization of the cell membrane failed to block or to elicit pulses. These results are interpreted as evidence that the neurones are functioning as conduction elements without conducted action potentials. The Cyanea system evidently has little in common with that of Nanomia, where all the evidence points to normal propagative electrogenesis. Considerable research into medusan neurophysiology over the last fifteen years suggests that scyphozoans and hydrozoans are profoundly different in the ways in which their behaviour is organized (L.M. PASSANO, this volume).

The question of the ionic basis of electrogenesis in Nanomia has been tentatively approached using sea waters made up with different ionic concentrations and by use of drugs. No clear picture emerges from this work. It will simply be noted here that tetrodotoxin, which selectively blocks the inward excitable channel for $\mathrm{Na}^{+}$in certain nerve preparations, had no effect on spike production in Nanomia. It was used at $10^{-6} \mathrm{~g} / \mathrm{ml}$ and specimens were kept in it for 5 hours. As will be seen from Figs. 1 and 2 the giant fibres are not exposed directly to the outside medium but are covered with a thin epithelium, so it is conceivable that the failure of the drug to work was due to its inability to penetrate into the region of the giant fibres. However squid axons are blocked at $10^{-8} \mathrm{~g} / \mathrm{ml}$, and it would be surprising if too little of the drug penetrated to have an appreciable effect. We may well therefore be dealing with a nervous system in which spike production is not sodium dependent. 
A critical experiment was to study the effects of cutting the giant fibres on conduction of the $\mathrm{F}^{1}$ and $\mathrm{F}^{2}$ events. If the giant fibres are the sole conductors of these events, sectioning them would abolish conduction. This is not found to be the case. The $\mathrm{F}^{1}$ and $\mathrm{F}^{2}$ signals continue to be recorded on the other side of the incision in their normal form and correct time relationships, essentially as if there had been no operation, except for a slight increase in conduction time which may or may not be significant. Clearly there are alternative routes through which impulses can spread following section of the two giants. Further, the fact that the operation does not lead to any breakdown in the independence of the two systems indicates that each giant fibre has its own alternative pathway. Conduction can still occur through a narrow bridge on the ventral side of the stem, representing about one quarter of the stem's thickness. This indicates that the alternative routes are distributed widely around the stem. While direct evidence of the nature of the alternative routes is lacking, there is a strong likelihood that they are to be found within the general ectodermal nerve net. Histologically the giant fibres have been envisaged as specializations within a dispersed nerve net, and such a model would explain the physiological results of sectioning the giant fibres, with the added requirement that the net be a double one, functionally speaking, even if this is not apparent at the level of gross microscopic anatomy.

In accordance with this picture, it may be noted that in the palpon tentacles of Nanomia there are two chains of nerve cells, one running up each side, without detected cross connections (Fig. 6A). Recordings from the tentacle show two fast conduction systems (Fig. 6B). Supposing the two chains to represent the two fast conducting systems, the tentacle can be taken as a simplified model for the stem, the main difference being that in the stem the two nerve nets have each become regionally specialized as giant fibres. There is some reason to suppose that this plan in its simpler form (two nets without giant fibres) occurs in calycophoran siphonophores as well as in some physonects, and that it is only in the long stemmed physonects like Nanomia, Forskalia, etc. that giant fibres have evolved. The tendency for formation of giant fibres appears to have gone furthest in Nanomia amongst known species. This is also suggested by study of the swimming bells; the ventral nerve tract which crosses the exumbrella is a bundle of small nerves in Forskalia (MACKIE, 1965), a single giant axon in Nanomia (Fig. 7).

The functional significance of double innervation in siphonophores probably lies in the gradation of muscle response intensity with distance from the site of stimulation. Both nervous systems appear to excite the same effectors in the same way. The only difference lies in the faster conduction rate of one of the two systems. Impulses initiated simultaneously in the two systems will be through conducted to all parts, but because of the conduction velocity difference a time interval of increasing duration will develop between the events the further they travel from the site of initiation. Recordings from the muscle tissue show that the magnitude of the muscle response 
is dependent on the interval between input signals, because of facilitation and temporal summation. The further apart the input signals, the weaker the response. Thus we have a mechanism permitting regulation of response intensity in relation to distance by frequency modulation of input signals from two synergistically acting excitatory systems. Evidence for this conclusion exists and will be presented elsewhere.
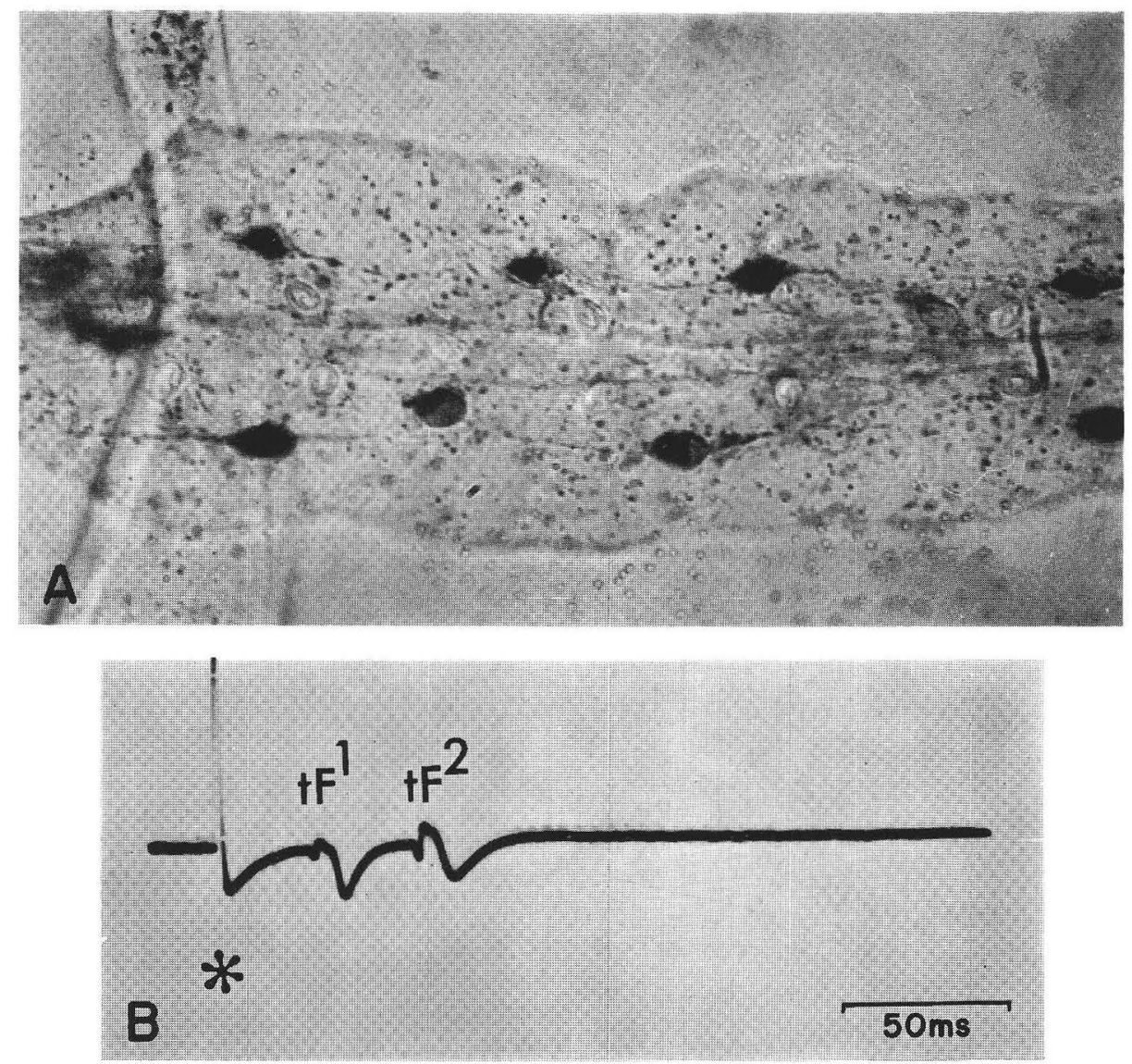

Fig. 6. Two nerve chains stained with methylene blue (a) and two fast conduction systems $\mathrm{tF}^{1}$ and $\mathrm{tF}^{2}(\mathrm{~B})$ in the palpon tentacle of Nanomia. Asterisk shows shock artefact in B, which is an extracellular recording.

\section{Discussion}

This paper is essentially an advance notice of work to be presented in more detail and with fuller documentation later. It contains many statements not backed up by evidence, which I ask the reader to excuse. The Nanomia preparation is a valuable one not least for the way in which it allows one to interpret extracellular recordings 


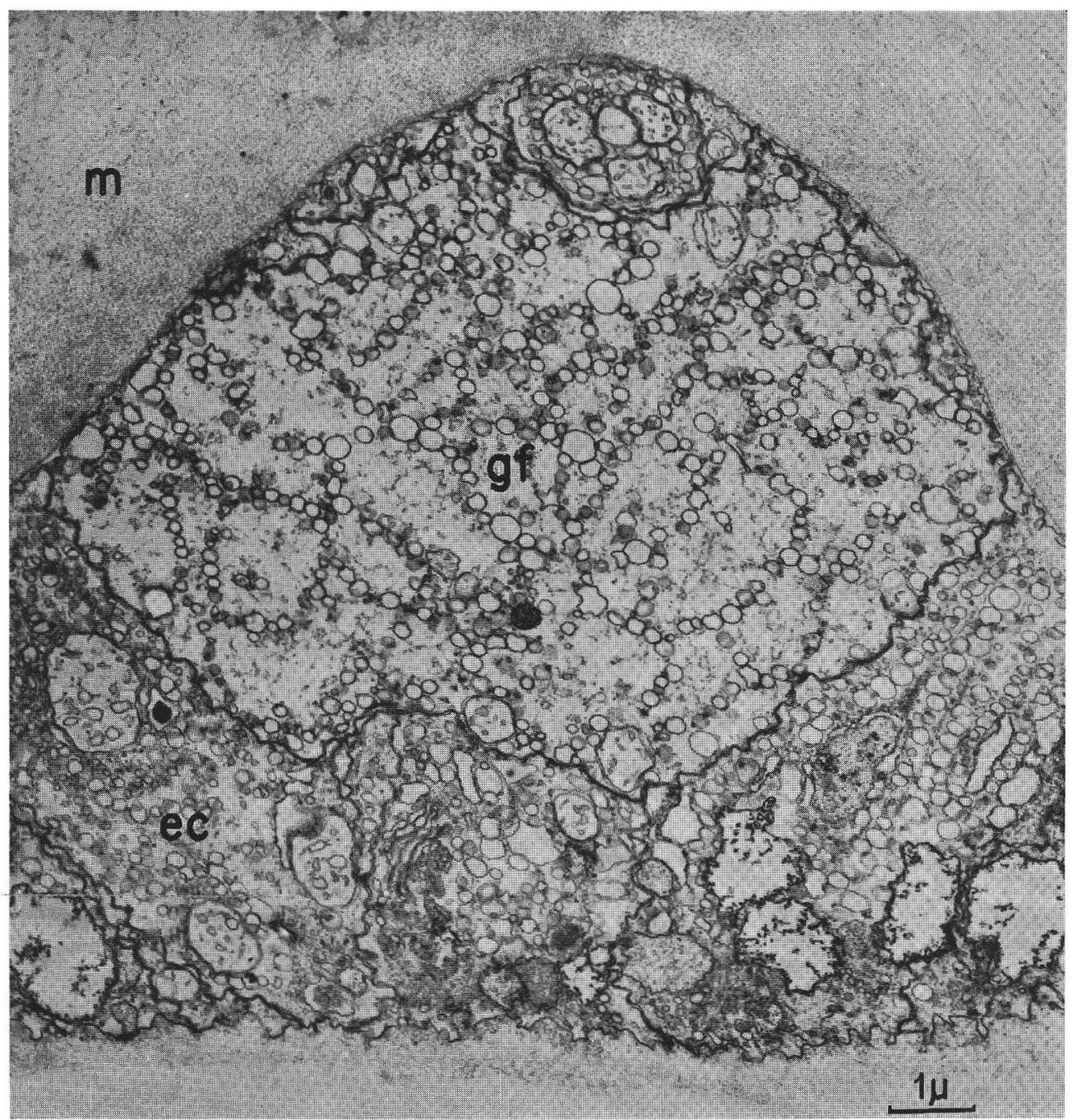

Fig. 7. A single giant fibre (gf) runs across the lower side of the swimming bell in Nanomia, conducting impulses for the forwards escape swimming response from the stem to the swimming centres in the bell margin. It is enveloped by processes of the ectoderm (ec) on all sides. $\mathrm{m}$, mesogloea.

and to show how signals recorded with suction electrodes can be broken down into a primary nervous component (constant wave-form and amplitude) and a secondary epithelial or epithelio-muscular component (wave-form and amplitude variable according to immediate past history). The preparation also allows analysis of fast and slow muscle contractions in terms of primary events. Without going into detail on this latter point, fast and slow contractions employ the same effectors but different conduction pathways, nervous in the former case, probably epithelial in the latter. 
There may be a parallel here with sea anemones (MACFARLANE, 1970).

The documentation presented in this paper centres around the identification of the giant axons as conductors of the two fast signal types, $F^{1}$ and $F^{2}$. The discovery of alternative physiological routes requires further histological attention, as we can now only infer that there are two routes through the general nerve net. It may be noted that Rushion (1945) obtained rather similar results in the earthworm, where he found that impulses reinvade an incised lateral giant fibre, presumably by proceeding through cross connections to the other lateral giant and back. This is only one of many parallels which may be drawn between the Nanomia giant fibre system and those of other invertebrates.

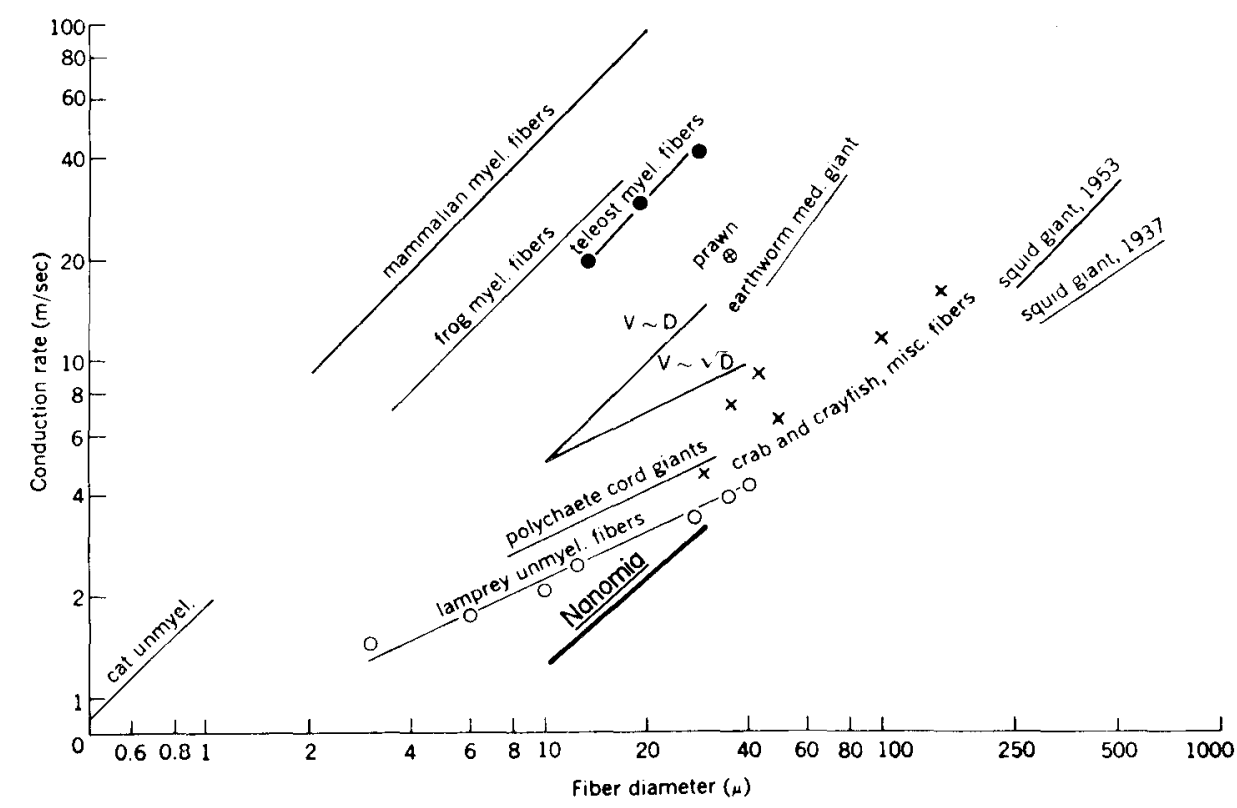

Fig. 8. Conduction velocities plotted against nerve fibre diameters for various animals, from Bullock and Horridge (1965) with the addition of new data from Nanomia. The Nanomia curve is taken from Fig. 4.

Fig. 8 shows a comparison of the Nanomia giant fibre system with those of other animals. Applying $\mathrm{V}=\mathrm{kd}$ (where $\mathrm{V}$ is the conduction velocity in $\mathrm{m} / \mathrm{sec}$ and $\mathrm{d}$ the diameter in microns) yields a $\mathrm{k}$ value of 0.1. As a measure of efficiency this places Nanomia above the squid $(\mathrm{k}=0.07-0.09)$ but below the earthworm $(\mathrm{k}=0.2-0.6)$ and most arthropods (0.1-0.6) (data summarized in BULLOCK and HorRIDGE, 1965). In theory (HoDGKIN, 1954) velocity should vary as the square root of fibre diameter, but as Fig. 8 shows, this is by no means always the case in practice, and it is not the case in Nanomia. 
The giant fibres in Nanomia are large by comparison with other coelenterate neurons, small compared with those in many invertebrates, but they appear to be averagely efficient. As SCHNEIDER (1892) first suggested, and as we can now demonstrate, they give the animal a capacity for fast response to stimulation which, lacking myelinated fibres, it could scarcely otherwise obtain. Their presence in Nanomia demonstrates once more that in spite of their low position on the evolutionary scale, coelenterates have essentially the same fundamental behavioural capabilities as other animals, which they develop as and where needed.

\section{REFERENCES}

Bullock, T. H. \& G. A. Horridge. 1965. Structure and Function in the Nervous Systems of Invertebrates. Freeman, San Francisco.

Hodgkin, A. L. 1954. A note on conduction velocity. J. Physiol., 125: 221-224.

KorotnefF, A. 1844. Zur Histologie der Siphonophoren. Mitt. Zool. Sta. Neapel. 5: 229-288.

MacFarlane, I. D. 1969. Two slow conduction systems in the sea anemone Calliactis parasitica. J. Exp. Biol., 51 : 377-385.

MACKIE, G. O. 1960. The structure of the nervous system in Velella. Quart. J. Micr. Sci., 101: 119-131.

MACKIE, G. O. 1964. Analysis of locomotion in a siphonophore colony. Proc. Roy. Soc. B., 159: 366-391.

MACKIE, G. O. 1965. Conduction in the nerve-free epithelia of siphonophores. Amer. Zool., 5: 439453.

Mackie, G. O. \& D. A. Boag. 1963. Fishing, feeding and digestion in siphonophores. Pubbl. Staz. Zool. Napoli, 33: 178-196.

Patton, M. L. \& L. M. Passano. 1972. Intracellular recording from the giant fiber nerve-net of a scyphozoan jellyfish. Amer. Zool., 12 (3): XXXV.

Rushton, W. A. H. 1945. Action potentials from the isolated nerve cord of the earthworm. Proc. Roy. Soc. B, 132: 423-437.

SCHAEPPI, T. 1898. Untersuchungen über das Nervensystem der Siphonophoren. Jena Zeit. Naturwiss., 32: 483-550.

SCHNeider, K. C. 1892. Einige histologische Befunde an Coelenteraten. Jena Zeit. Naturwiss., 27: $387-461$.

SPenCER, A. N. 1971. Myoid conduction in the siphonophore Nanomia bijuga. Nature, 233: 490-491.

\section{DISCUSSION}

Ross: Did you detect any threshold differences between the two conducting systems?

MACKIE: I found no consistent threshold differences.

Passano: What velocities do you record for the $\mathrm{F}^{1}$ and $\mathrm{F}^{2}$ conducting systems from the Nanomia tentacle?

MACKIE: The velocities are much slower than in the stem—in the order of $35-50 \mathrm{~cm} / \mathrm{sec}$. 